Notre Dame Journal of Formal Logic

Volume 48, Number 2, 2007

\title{
A Note on Weakly O-Minimal Structures and Definable Completeness
}

\begin{abstract}
Alfred Dolich
Abstract We consider the extent to which certain properties of definably complete structures may persist in structures which are not definably complete, particularly in the weakly o-minimal structures.
\end{abstract}

\section{Introduction}

In this short note we study weakly o-minimal theories and how they relate to general ordered theories which are not definably complete. First, we consider the degree to which topological properties of definable sets in weakly o-minimal structures mirror those in o-minimal structures. Second, we consider the degree to which weakly o-minimal theories may be characterized as the "best-behaved," densely ordered theories among those theories which are not definably complete. Here we are motivated by results characterizing o-minimal theories as those definably complete theories bearing certain desirable properties.

For the problems we consider that our answers are negative. Recall the definition of weak o-minimality.

Definition 1.1 A structure $(M,<, \ldots)$ in a language $\mathcal{L}$ with a symbol $<$ for a dense linear order is called weakly o-minimal if any definable $X \subseteq M$ is a finite union of convex sets. A theory $T$ is weakly o-minimal if all of its models are. (See, for example, [8] and the references therein.)

Also recall the definition of definable completeness (for a discussion of this, see [15]).

Definition 1.2 A structure $(M,<, \ldots)$ in a language $\mathcal{L}$ with a symbol $<$ for a dense linear order is said to be definably complete if, for any definable subset $X \subseteq M$, if $X$ is bounded above then there is a supremum $a \in M$ of $X$. Similarly, we demand

Received November 11, 2005; accepted October 16, 2006; printed May 14, 2007

2000 Mathematics Subject Classification: Primary, 03C64; Secondary, 03C52

Keywords: o-minimal, weakly o-minimal

(c) 2007 University of Notre Dame 
there be an infimum if $X$ is bounded below. Note that if $M$ is definably complete and $N \equiv M$ then $N$ is definably complete. Hence we say a theory $T$ is definably complete if all (or equivalently one) of its models is definably complete.

Recall that any o-minimal theory is definably complete (see [15]). Also notice that if $M$ is weakly o-minimal but not o-minimal then definable completeness must fail for $M$. Recall that, in analogy to o-minimal theories, weakly o-minimal structures or theories satisfy many desirable properties (see [8]). Hence we are led to our initial question, to what extent is weak o-minimality an appropriate analogue for o-minimality in the class of theories which are not definably complete?

In Sections 2 and 3 we consider two questions. First, we consider a topological property, namely, whether given $M$ weakly o-minimal and $X \subseteq M^{n+1}$ definable, closed, and bounded, it is necessarily the case that $\pi(X)$ is closed where $\pi\left(M^{n+1}\right) \rightarrow M^{n}$ is a coordinate projection. Note that it follows from facts in [11] that this is true when $M$ is o-minimal. Our work shows that the answer to this question is negative, but in specific cases the desired property may still hold. Hence from this point of view we may not characterize weakly o-minimal theories as those theories which are not definably complete yet have desirable topological properties. Second, we focus on results concerning theories expanding densely ordered groups satisfying definable completeness which allow one to conclude that under various conditions the theory is o-minimal or "close" to o-minimal. We ask whether we may remove the assumption of definable completeness if we weaken the conclusion to the analogous statement with "weakly o-minimal" in place of "o-minimal." Our result is negative and Section 3 is devoted to counterexamples.

\section{Projections of Closed Bounded Sets}

Throughout this section, given a structure $M$, we will call a function $\pi$ a projection if it is a coordinate projection between Cartesian powers of $M$. As mentioned in Section 1, if $T$ is o-minimal, $M \models T, X \subseteq M^{n}$ is closed bounded and definable, and $\pi$ is a projection function from $M^{n}$, then $\pi(X)$ is closed. In [11] it is shown that this holds in any definably complete structure. We explore the extent to which the converse of this result holds. We focus primarily on the case of weakly o-minimal structures.

Proposition 2.1 Let $\mathcal{M}=\langle M,+,<, \ldots\rangle$ be an Archimedean model of the theory of densely ordered Abelian groups. Then the following are equivalent:

1. $\mathcal{M}$ is definably complete;

2. if $X \subseteq M^{n}$ is a closed bounded definable set and $\pi$ is any projection then $\pi(X)$ is closed.

Note that since properties (1) and (2) above are elementary, the condition that $M$ is Archimedean may be weakened to the assumption that $M$ is elementarily equivalent to $N$ which is Archimedean.

Proof (1) implies (2) was proved in greater generality by C. Miller in [11].

(2) implies (1) To establish that $M$ is definably complete it suffices via results from [11] to show that if $U \subseteq M$ is definable, open, closed, and nonempty then $U=M$. Fix $U$ open, closed, and nonempty. Without loss of generality, we may assume that $0 \in U$. Let $W$ be the convex component of $U$ containing 0 . Note that $W$ is closed 
and definable; we show that $W=M$. Fix some element $d>0$ so that $-d \in W$. Consider the following set:

$$
X:=\{(x, y) \in[0, d] \times W: y \geq-d \& x+y \notin W\}
$$

Let $\pi(X)$ be the projection of $X$ onto the first coordinate. Consider the following facts about $X$ and $\pi(X)$ :

1. $X$ is closed;

2. $0 \notin \pi(X)$;

3. $(0, d] \subseteq \pi(X)$.

The first two are clear from the definition of $X$. For the third fact, fix $\epsilon \in(0, d]$; if $\epsilon \notin \pi(X)$ then for any $e \in W$ we must have that $e+\epsilon \in W$, which is impossible since $M$ is Archimedean. Thus under our assumption we must have that $X$ is not bounded, and hence that $W$ is not bounded above. We may argue symmetrically to get that $W$ is not bounded below. Hence $W=M$.

The structure $\langle\mathbb{Q},+,<,(-\sqrt{2}, \sqrt{2})\rangle$ is an example of a weakly o-minimal structure (for the weak o-minimality, see [1]) of the type considered in Proposition 2.1, and hence there are closed bounded definable sets $X$ and projections $\pi$ so that $\pi(X)$ is not closed.

The following two propositions give simple examples to show that several assumptions in Proposition 2.1 are necessary. The following lemma is elementary; we provide a statement and brief proof for completeness and to emphasize the exact statement we need.

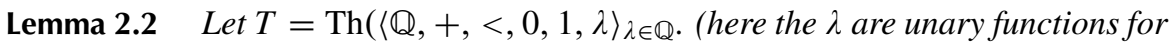
multiplication by $\lambda$ ). Let $M \models T$ and $X \subseteq M^{n}$ be a definable set. Then $X$ can be written as the union of definable sets $X_{i}$ where each $X_{i}$ is defined by a formula $\varphi(\bar{x})$ of the form,

$$
\left(A_{1} \wedge \cdots \wedge A_{n}\right)
$$

Here each $A_{i}$ is of the form $t_{1}<t_{2}$ or $t_{1}=t_{2}$ for $t_{1}, t_{2}$ terms. These terms may contain the same parameters used to define $X$. Furthermore, the closure of $X_{i}$ is definable via a formula obtained by replacing in $\varphi$ each $A_{i}$ of the from $t_{1}<t_{2}$ by $t_{2} \leq t_{2}$. An analogous result holds for $\langle\mathbb{Q},<, 0\rangle$.

Proof This is a simple exercise in cell decomposition using the fact that $T$ has quantifier elimination.

We also need a simple topological fact.

Fact 2.3 Let $X$ be a topological space. If $A \subseteq X, U \subseteq X$, and $U$ is both closed and open then $\operatorname{cl}(A \cap U)=\operatorname{cl}(A) \cap U$.

We show that the assumption of $M$ being Archimedean in Proposition 2.1 is necessary. We begin by pointing out that, in Proposition 2.1, in order to establish that (2) implies (1) we may replace the assumption that $M$ is Archimedean by

There is a pair of open convex sets $U$ and $V$ such that $U \cup V=M$, for all $x, y$ if $x \in U$ and $y \in V$ then $x<y$, and there is no $\epsilon>0$ such that for all $x \in U$ we also have that $x+\epsilon \in U$.

In the terminology of [8], this condition is met if $M$ is of nonvaluational type. We show that this is necessary. 
Proposition 2.4 Let $\mathcal{R}=\langle\mathbb{R} \times \mathbb{Q},<,+,(0,0),(0,1), U, \lambda\rangle_{\lambda \in \mathbb{Q}}$ be a structure for the language $\{<,+, 0,1, U, \lambda\}_{\lambda \in \mathbb{Q}}$. In $\mathcal{R}$, $<$ is lexicographic ordering, $+i$ is componentwise addition, $U:=\{0\} \times \mathbb{Q}$, and $\lambda$ is componentwise multiplication by the rational number $\lambda$. If $X \subseteq R^{n}$ is a closed set and $\pi$ is any projection then $\pi(X)$ is closed.

Proof Throughout the proof we let $\mathscr{L}$ be the language $\{<,+, 0,1, \lambda\}_{\lambda \in \mathbb{Q}}$ and write $\mathscr{L}(R)$ for the language $\mathscr{L}$ expanded by constants for the universe of $\mathcal{R}$. We first establish that the theory eliminates quantifiers. After some simple manipulation and omitting easy cases this reduces to two main cases. First, we eliminate the existential quantifier from a formula of the form,

$$
\varphi:=\exists y\left(t_{0}<y<t_{1} \wedge \bigwedge_{j=1}^{M} \neg U\left(\lambda_{j} y+r_{j}\right)\right),
$$

where $t_{0}, t_{1}$, and $r_{i}$ are all terms in which $y$ does not appear. This formula is equivalent to the conjunction of

$$
U\left(t_{0}-t_{1}\right) \rightarrow t_{0}<t_{1} \wedge \bigwedge_{j=i}^{M} \neg U\left(\lambda_{j} t_{0}+r_{j}\right)
$$

and

$$
\neg U\left(t_{0}-t_{1}\right) \rightarrow t_{0}<t_{1} .
$$

The equivalence of $\varphi$ and this conjunction follows from these two facts:

1. $\mathcal{R} \models \forall x \forall y \forall z(U(x-y) \rightarrow(U(\lambda x+z) \leftrightarrow(U \lambda y+z))$ for all $\lambda$;

2. $\mathcal{R} \models \forall x \forall y \forall z_{1} \ldots \forall z_{n}((\neg U(x-y) \wedge x<y) \rightarrow$

$$
\left.\exists w\left(x<w<y \wedge \bigwedge_{i=1}^{n} \neg U \lambda_{i} w+z_{i}\right)\right) \text { for all } n \text { and } \lambda_{1} \ldots \lambda_{n} .
$$

The first fact is immediate; the second follows since the set $U$ interpreted in $\mathcal{R}$ is countable while if $a, b \in \mathcal{R}$ are such that $\mathcal{R} \models a\langle b \wedge \neg U(a-b)$ then the interval $(a, b)$ in $\mathcal{R}$ is uncountable.

In the second case, we must eliminate the quantifier from

$$
\exists y\left(t_{0}<y<t_{1} \wedge \bigwedge_{i=1}^{N} U\left(\lambda_{i} y+s_{i}\right) \wedge \bigwedge_{j=1}^{M} \neg U\left(\lambda_{j} y+r_{j}\right),\right.
$$

where the $t \mathrm{~s}, r \mathrm{~s}$, and $s \mathrm{~s}$ are terms in which $y$ does not appear. This is equivalent to

$$
\exists y\left(t_{0}<y<t_{1} \wedge U \lambda_{1} y+s_{1} \wedge \bigwedge_{i=2}^{N} U\left(\lambda_{1}^{-1} s_{1}-\lambda_{i}^{-1} s_{i}\right) \wedge \bigwedge_{j=1}^{M} \neg U\left(\lambda_{1}^{-1} s_{1}-\lambda_{j}^{-1} r_{j}\right)\right) .
$$

This follows since

$$
\mathcal{R} \models \forall x \forall y \forall z\left(\left(U\left(\lambda_{1} x+z\right) \wedge U\left(\lambda_{2} x+y\right)\right) \leftrightarrow\left(U\left(\lambda_{1} x+z\right) \wedge U\left(\lambda_{1}^{-1} z-\lambda_{2}^{-1} y\right)\right)\right) .
$$

So we are reduced to considering

$$
\exists y\left(t_{0}<y<t_{1} \wedge U\left(\lambda_{1} y+s_{1}\right)\right) .
$$

This is readily seen to be equivalent to

$$
\begin{aligned}
& t_{0}<t_{1} \wedge\left(U\left(\lambda_{1} t_{0}+s_{1}\right)\right. \vee U\left(\lambda_{1} t_{1}+s_{1}\right) \vee \\
&\left.\left(\lambda_{1} t_{0}+s_{1}<0<\lambda_{1} t_{1}+s_{1}\right) \vee\left(\lambda_{1} t_{1}+s_{1}<0<\lambda_{1} t_{0}+s_{1}\right)\right) .
\end{aligned}
$$


Hence we have quantifier elimination. To verify the property on projections, we fix $X \subseteq R^{n+1}$ closed and definable. We show that the projection of $X$ onto the first $n$ coordinates is closed. By quantifier elimination, Lemma 2.2, and Fact 2.3, we may assume $X$ is defined by a formula $\varphi(\bar{x})$ of the form,

$$
\psi\left(x_{1}, \ldots, x_{n+1}\right) \wedge \bigwedge_{i \in I} U\left(t_{i}\right) \wedge \bigwedge_{j \in J} \neg U\left(t_{j}\right),
$$

where the $t_{i}$ s and $t_{j}$ s are terms in $\mathcal{L}(R), I$ and $J$ are disjoint finite sets of indices, and $\psi$ is a formula in $\mathcal{L}(R)$ of the form,

$$
\left(A_{1} \wedge \cdots \wedge A_{m}\right),
$$

with each $A_{i}$ of the form $r_{1} \leq r_{2}$ or $r_{1}=r_{2}$ for terms $r_{1}$ and $r_{2}$. After some simple manipulation we are reduced to showing formulas of the form,

$$
\exists x_{n+1}\left(t_{1} \leq x_{n+1} \leq t_{2} \wedge \bigwedge_{i \in I} U s_{i} \wedge \bigwedge_{j \in J} \neg U s_{j}\right),
$$

define closed sets, where $t_{1}$ and $t_{2}$ are terms in $\mathcal{L}(R)$ not containing $x_{n+1}$, the $s_{i}$ s and $s_{j}$ s are terms in $\mathscr{L}(R)$, and $I$ and $J$ are disjoint finite sets of indices. Assuming that $I$ is nonempty, we may argue as in the proof of quantifier elimination to reduce to that case of showing that a formula of the form,

$$
\exists x_{n+1}\left(t_{1} \leq x_{n+1} \leq t_{2} \wedge U\left(\lambda x_{n+1}+s\right)\right),
$$

where $t_{1}, t_{2}$, and $s$ are $\mathcal{L}(R)$ terms, defines a closed set. But applying the quantifier elimination outlined above this formula is equivalent to

$$
\begin{aligned}
& t_{1} \leq t_{2} \wedge\left(U\left(\lambda t_{1}+s\right) \vee U\left(\lambda t_{2}+s\right)\right. \vee \\
&\left.\left(\lambda t_{1}+s \leq 0 \leq \lambda t_{2}+s\right) \vee\left(\lambda t_{2}+s \leq 0 \leq \lambda t_{1}+s\right)\right)
\end{aligned}
$$

which is closed. The case when $J$ is empty is similar.

Note that by [1] the above structure is weakly o-minimal.

We now show that in Proposition 2.1 the assumption that the structure expands that of an ordered group is, in essence, necessary. Notice that the structure being "Archimedean" as normally understood does not apply in this context. Here we give an example with universe $\mathbb{Q}$ which is Archimedean in the sense discussed in [9].

Proposition 2.5 $Q Q Q=\langle\mathbb{Q},<, P, 0\rangle$, the ordered rationals with a predicate $P$, for the interval between $-\infty$ and $\pi$, has the property that projections of closed definable sets are closed.

Proof The proof is elementary and similar to that of the previous proposition; we provide a sketch. We let $\mathcal{L}$ be the language $\{<, 0\}$ and let $\mathscr{L}(\mathcal{Q})$ be $\mathcal{L}$ augmented by constants for the rational numbers. First, notice that $\operatorname{Th}(\mathcal{Q})$ eliminates quantifiers. (Given a formula of the form $\exists x \varphi(x, \bar{y})$ with $\varphi$ quantifier-free, one can easily write out an equivalent quantifier-free formula.) Let $X \subseteq \mathbb{Q}^{n+1}$ be a closed Q-definable set. We show that the projection of $X$ onto the first $n$ coordinates is closed. By the quantifier elimination for $\operatorname{Th}(\mathcal{Q})$, Lemma 2.2, and Fact 2.3, we may reduce to the case where $X$ is defined by a formula $\varphi\left(x_{1}, \ldots, x_{n+1}\right)$ of the form,

$$
\psi\left(x_{1}, \ldots, x_{n+1}\right) \wedge \bigwedge_{i \in I} P t_{i} \wedge \bigwedge_{j \in J} \neg P t_{j},
$$


where the $t_{i}$ s and $t_{j} s$ are $\mathcal{L}(\mathcal{Q})$-terms, $I$ and $J$ are disjoint finite sets of indices, and $\psi$ is a quantifier-free $\mathscr{L}(\mathcal{Q})$-formula with no strict inequalities appearing. Furthermore, $\psi$ has the form,

$$
\left(A_{1} \wedge \cdots \wedge A_{n}\right)
$$

where each $A_{i}$ is of the form $s_{1} \leq s_{2}$, or $s_{1}=s_{2}$ for some $\mathcal{L}(\mathcal{Q})$-terms $s_{1}$ and $s_{2}$. The fact that $\exists x_{n+1} \varphi(\bar{x})$ defines a closed set follows immediately from the quantifier elimination as in Proposition 2.4.

Note that by the main result in [1] the above structure is weakly o-minimal.

We end this section by pointing out that our results contrast oddly with results in [8], where it is shown that if $M$ is a weakly o-minimal structure of nonvaluational type then it should be considered to behave very similarly to an o-minimal structure. Here we show that having nonvaluational type is exactly a condition that allows us to verify that the projection of a closed bounded definable set is not closed, in direct contrast to the o-minimal case. On the other hand, we show that there are structures of valuational type where the desired property of projections does hold.

\section{Examples}

In this section we wish to consider whether there are weakly o-minimal analogues of two facts relating definable completeness and o-minimality. We begin with some necessary definitions.

Definition 3.1 Given a theory $T$ we say that $\mathrm{T}$ satisfies uniform finiteness if, for any formula $\varphi(x, \bar{y})$, there is a natural number $N \in \mathbb{N}$ so that if $M \models T, \bar{a} \in M$, and $\varphi(M, \bar{a})$ is finite, then $\varphi(M, \bar{a})$ has size at most $N$.

Definition 3.2 Given a topological space $A, X \subseteq A$ is called constructible if it is a finite Boolean combination of open sets.

Definition 3.3 Let $M$ be a structure for a language including a symbol $<$ so that $<$ is interpreted as a dense linear order in $M$. The open core of $M$, denoted $M^{\circ}$, is the reduct of $M$ generated by all of the definable open sets. (See, for example, [12].)

We consider the following two facts. The first follows easily from facts in Section 2 of [12] combined with results from [5]. The second is from [4].

Fact 3.4 If $M$ is a model of some definably complete expansion of the theory of densely ordered groups such that any definable $X \subseteq M$ is constructible and there is no definable $X \subseteq M$ which is infinite and discrete, then $M$ is o-minimal.

Fact 3.5 If $T$ is some expansion of the theory of densely ordered groups which satisfies uniform finiteness and is definably complete, then if $M \models T, M^{\circ}$ is ominimal.

The goal of this section is to consider analogues of the above statements with the assumption of definable completeness dropped. In order to formulate reasonable analogues of the above in the absence of definable completeness we have to replace the assumption that $M$ is an expansion of the theory of densely ordered groups with the assumption that $M$ is an expansion of divisible ordered Abelian groups (by results in [11] if $M$ is definably complete and a densely ordered group then it is also divisible and Abelian). Given this we ask 
1. if $M$ is a model of some expansion of the theory of divisible ordered Abelian groups such that any definable $X \subseteq M$ is constructible and there is no definable $X \subseteq M$ which is infinite and discrete, must $M$ be weakly o-minimal?

2. if $T$ is some expansion of the theory of divisible ordered Abelian groups which satisfies uniform finiteness, then, if $M \models T$, must $M^{\circ}$ be weakly ominimal?

We construct a single example to refute both of the above statements. The example shows that even if we add the condition that $T$ satisfy the exchange property for definable closure the conclusion does not follow. Also our example will be a model of the theory of real closed fields, so assuming that we work with expansions of the theory of real closed fields rather than of densely ordered groups still does not yield a true statement.

We point out that the motivation for our example comes from [2], where the idea of adding a new random set to a structure is introduced. In our case we desire, rather than adding a truly random set, to add a new random open set. This should be made clear in the ensuing construction.

Let $T_{\mathrm{RCF}}$ be the theory of real closed fields formulated in language $\mathscr{L}_{\mathrm{df}}$ where every definable function is given by a term in the language, so that $T_{\mathrm{RCF}}$ eliminates quantifiers and is universally axiomatizable. Fix $\mathcal{R}$ a sufficiently saturated model of $T_{\mathrm{RCF}}$. Let $V$ be a nontrivial convex subring of $\mathcal{R}$, and let $\mathcal{L}_{\text {conv }}$ be $\mathcal{L}_{\text {df }}$ augmented with a unary predicate for $V$. Let $(\mathcal{R}, V)$ be $\mathcal{R}$ formulated in $\mathscr{L}_{\text {conv }}$ in the obvious way, so $(\mathcal{R}, V)$ is a real closed valued field. Then we have the following fact.

Fact 3.6 (van den Dries and Lewenberg in [7], Cherlin and Dickmann in [3]) $T_{\text {conv }}=\operatorname{Th}((\mathcal{R}, V))$ eliminates quantifiers in $\mathscr{L}_{\text {conv }}$ and is weakly o-minimal. $T_{\text {conv }}$ may be axiomatized by stating that $\mathcal{R}$ is a real closed field and that $V$ is a nontrivial convex subring.

We need a fact about $T_{\text {conv }}$ which can be found in [6].

Fact 3.7 $T_{\text {conv }}$ satisfies the exchange property for definable closure.

Following Mellor (see [10]) we may also think of a $T_{\text {conv }}$ structure formulated in a three-sorted language $\mathscr{L}_{3}$ with sorts,

1. $(\mathcal{R},+,-, \cdot, 0,1,<)$,

2. $(\Gamma,+,-, 0, \infty,<)$,

3. $(k,+,-, \cdot, 0,1,<)$,

where $\Gamma$ is the value group and $k$ is the residue field.

We also need to add functions,

1. $v: \mathcal{R} \rightarrow \Gamma$,

2. Res : $\mathcal{R}^{2} \rightarrow k$.

We let $T_{3}$ be the $\mathscr{L}_{3}$ theory stating

1. $\mathcal{R}$ is a real closed field,

2. $\Gamma \backslash \infty$ is a divisible ordered Abelian group,

3. $k$ is a real closed field,

4. $v$ is the valuation map,

5. Res is the map such that $\operatorname{Res}(x, y)=x y^{-1}$ if $v(x) \geq v(y)$ and 0 otherwise.

Any model $M$ of $T_{\text {conv }}$ may also be considered as a model of $T_{3}$; we will denote the associated $\mathscr{L}_{3}$-structure $M_{3}$. 
Fact 3.8 (Mellor in [10]) $\quad T_{3}$ eliminates quantifiers in $\mathscr{L}_{3}$.

The above theorem implies the following corollary already noted in [7].

Corollary 3.9 If $M \models T_{3}$, the induced structure on the group sort is just the divisible Abelian group structure, whereas that on the residue field sort is just the real closed field structure.

Given $M=T_{3}$, let $M_{\text {conv }}$ be the structure whose underlying set is the first sort in $M$ formulated in the language $\mathcal{L}_{\text {conv }}$. Note that $M_{\text {conv }} \models T_{\text {conv }}$.

We need a simple fact about $T_{3}$.

Lemma 3.10 $\quad T_{3}$ satisfies uniform finiteness.

Proof This is immediate from the fact that o-minimal and weakly o-minimal theories satisfy uniform finiteness.

Given this we now may augment $\mathcal{L}_{3}$ with a new predicate $G$ to form $\mathcal{L}_{3}^{G}$. Fix $\widehat{M}=T_{3}$, sufficiently saturated, we augment $\widehat{M}$ to an $\mathcal{L}_{3}^{G}$ structure $\widehat{M}^{G}$ by adding a "random" subset to the value group sort as in [2]. We let $T_{3}^{G}$ be the theory of $\widehat{M}^{G}$.

Working in $\widehat{M}^{G}$, we let $\widehat{M}_{\text {conv }}^{G}$ be the valued field sort formulated in the language $\mathscr{L}_{\text {conv }}^{G}:=\mathscr{L}_{\text {conv }} \cup\{H\}$ with a new unary predicate. In $\widehat{M}_{\text {conv }}^{G}$ set

$$
H\left(\widehat{M}_{\text {conv }}^{G}\right):=\left\{x: \widehat{M}^{G} \models G v(x)\right\} .
$$

Let $T_{\text {conv }}^{G}=\operatorname{Th}\left(\widehat{M}_{\text {conv }}^{G}\right)$

As above, given $M \models T_{\text {conv }}^{G}$, we let $M_{3}$ be the associated $\mathscr{L}_{3}^{G}$-structure, setting

$$
G\left(M_{3}\right):=\{x \in \Gamma: M \models G y \text { for any } y \text { such that } v(y)=x\} .
$$

We note an easy fact.

Lemma 3.11 If $M \models T_{\text {conv }}^{G}$, then $M_{3} \models T_{3}^{G}$.

Proof $\quad$ If $M=T_{\text {conv }}^{G}$, then $M \equiv \widehat{M}_{\text {conv }}^{G}$ by definition. Also $M^{\text {eq }} \equiv \widehat{M}_{\text {conv }}^{G \text { eq }}$. But then we see that $M_{3} \equiv \widehat{M}$, and hence $M_{3} \models T_{3}^{G}$.

We can now list the relevant facts about $T_{\text {conv }}^{G}$.

Theorem 3.12 Let $M \models T_{\text {conv }}^{G}$; then

1. $H(M)$ is open and has infinitely many convex components,

2. any definable $X \subseteq M$ is constructible,

3. $T_{\mathrm{conv}}^{G}$ satisfies the exchange property for $\mathrm{dcl}$,

4. there are no definable $X \subseteq M$ which are infinite and discrete,

5. $T_{\mathrm{conv}}^{G}$ satisfies uniform finiteness.

\section{Proof}

1 For (1) simply note that, for any $x$ in the value group of $M_{3}, v^{-1}(x)$ is open. By the genericity of $G$ for any $x, y \in G, v^{-1}(x)$ and $v^{-1}(y)$ are disjoint sets.

2 Let $X \subseteq M$ be any definable set. Note that $X$ is also a definable set in $M_{3}$; hence it suffices to show that any definable subset of the first sort in $M_{3}$ is constructible. 
By the quantifier elimination for $T_{3}$ and properties of the generic extension (see [2]) a definable subset of the valued field sort is of the form,

$$
\varphi(x) \wedge \bigwedge_{i=1}^{N} G_{i} f(x) \wedge \bigwedge_{j=1}^{M} \neg G g_{j}(x),
$$

where $\varphi(x)$ is a formula in $\mathscr{L}_{3}$ and the $f \mathrm{~s}$ and $g \mathrm{~s}$ are $T_{3}$-definable functions from the valued field sort to the value group. For constructibility it thus suffices to show that if $f$ is a $T_{3}$-definable function from the first sort to the value group then $f^{-1}(G)$ is constructible (that $f^{-1}(\neg G)$ is constructible will be identical). Hence suppose we have a formula $\psi(x, y, \bar{z})$ such that $x$ is a variable in the value group sort and that for parameters $\bar{c}$ and an element $b$ in the valued field sort, $\psi\left(M_{3}, b, \bar{c}\right)$ is finite. Without loss of generality, we may assume this definable set has cardinality one. A simple argument using quantifier elimination yields that if $M_{3} \models \psi(a, b, \bar{c})$ then there are finitely many definable functions $g_{1}, \ldots, g_{n}$ from $\Gamma^{N} \rightarrow \Gamma$ (for some $N$ ), and finitely many definable functions $h_{1}, \ldots, h_{m}$ from $M$ to $M^{N}$ so that for any $x$ in the domain of $f, f(x)=g_{i}\left(v\left(h_{j}(x)\right)\right)$ for some $i, j$. Thus it suffices to show that, for any $h: M \rightarrow M^{N}$ definable and $g: \Gamma^{N} \rightarrow \Gamma$ definable, the set $h^{-1}\left(v^{-1}\left(g^{-1}(G)\right)\right)$ is constructible. But note that $v^{-1}\left(g^{-1}(G)\right)$ is open. Then by the weak o-minimality of $T_{\text {conv }}$ and the $T_{3}$-definability of $h, h^{-1}\left(v^{-1}\left(g^{-1}(G)\right)\right)$ is constructible.

3 It suffices to show that if $a \in \operatorname{dcl}(\bar{b})$ in $M$ then $a \in \operatorname{dcl}_{T_{\text {conv }}}(\bar{b})$; that is, the definable closure relation in $M$ is the same as that in its restriction to $\mathscr{L}_{\text {conv }}$. Exchange then follows from Fact 3.7 .

Suppose that $a \in \operatorname{dcl}(\bar{b})$ in $M$. Then working in $M_{3}$ we also have that $a \in \operatorname{dcl}(\bar{b})$ in $M_{3}$, which is most easily seen by realizing that $M_{3}$ is interdefinable with a finite set of sorts from $M^{\text {eq }}$. But by the genericity of the set $G, a \in \operatorname{dcl}_{T_{3}}(\bar{b})$, that is, thinking of $M_{3}$ as an $\mathscr{L}_{3}$ structure. But then we see that $a \in \operatorname{dcl}_{T_{\text {conv }}}(\bar{b})$ in $M$ thought of as an $\mathscr{L}_{\text {conv }}$ structure (once again since $M_{3}$ is a fragment of $M^{\text {eq }}$ ).

4 Assertion (4) follows easily from (3). Suppose that there were $X \subseteq M$ infinite, discrete, and definable with parameters $\bar{a}$. Without loss of generality we may assume that $M$ is $\omega$-saturated. Hence there is $e \in X \backslash \operatorname{dcl}(\bar{a})$. Since $X$ is discrete there is an open interval $I$ so that $I \cap X=\{e\}$. Now note that if $d \in I$ and $d<e$ then $e \in \operatorname{dcl}(d \bar{a})$ as witnessed by the formula $\varphi(y)$,

$$
d<y \wedge y \in X \wedge \forall z(z \in X \wedge d<z \rightarrow y \leq z) .
$$

But by the saturation of $M$ there is $d \in I$ with $d<e$ so that $d \notin \operatorname{dcl}(e \bar{a})$. Hence exchange would fail.

5 Assertion (5) follows from (4) since if uniform finiteness were to fail there would be an infinite discrete $X$ definable in some model of $T_{\text {conv }}^{G}$.

To finish this section we briefly point out that a much simpler example of the above behavior can be obtained if we do not demand an expansion of a real closed field. Furthermore, we obtain an example without the independence property.

Let $\mathcal{L}=\{+,<, c, \lambda\}_{\lambda \in \mathbb{Q}}$ where the $\lambda$ are unary function symbols. We let $\mathcal{L}^{*}=\mathscr{L} \cup\{H, V\}$ where $H$ and $V$ are unary predicates symbols. Let $\mathcal{Q}$ be the $\mathscr{L}^{*}$ structure with universe $\mathbb{R} \times \mathbb{Q}$ where + is interpreted as componentwise addition and $<$ is interpreted as lexicographic order. Furthermore, we interpret each $\lambda$ as 
componentwise multiplication by $\lambda$ and $c$ as $(0,0)$. Finally, we interpret $H$ as the set $\mathbb{Q} \times \mathbb{Q}$ and $V$ as $\{0\} \times \mathbb{Q}$. Let $T=\operatorname{Th}(\mathcal{Q})$.

Proposition 3.13 Thas quantifier elimination.

Proof This is essentially the same as the proof in Proposition 2.4.

Proposition 3.14 Let $M \models T$; then the analogues of (1)-(5) of Theorem 3.12 hold.

Proof (1) is obvious and (2) follows from quantifier elimination since any set definable by an atomic formula is constructible. As in Theorem 3.12, (4) and (5) follow from (3). For (3) we show that if $X \subset M$ is finite and definable with parameters from $A \subset M$, then $X \subseteq \theta(M)$ for some $\mathcal{L}$-formula $\theta$ with parameters from $A$ so that $\theta(M)$ finite. Then the exchange property follows by the o-minimality of $M 「_{\mathscr{L}}$ and the fact that o-minimal structures satisfy exchange (see [15]). Hence suppose that $\varphi(x)$ is a formula with parameters from $A$ which defines a nonempty finite set. Applying quantifier elimination and eliminating simple cases we are reduced to considering $\varphi$ of the form,

$$
\psi(x) \wedge \bigwedge_{i=1}^{N} P_{i} t_{i} \wedge \bigwedge_{j=1}^{M} \neg Q_{j} s_{j},
$$

where $P_{i}, Q_{j} \in\{H, V\}$, the $s_{i}, t_{j}$ s are terms, and $\psi(x)$ is an $\mathcal{L}$-formula with parameters from $A$. By o-minimality of $M \uparrow_{\mathscr{L}}$ we may assume that $\psi(M)$ is a point or an interval. If $\psi(M)$ is a single point then we are done; hence assume that $\psi(M)$ is an interval. Then $\varphi(M)$ would have interior since $H$ and $V$ define sets which are both open and closed and terms define global continuous functions. But this contradicts that $\varphi(M)$ is finite, and we have our desired result.

Proposition 3.15 $T$ does not have the independence property.

Proof Fix $M \models T$ highly saturated and let $\left\{a_{i}: i \in \omega\right\}$ be an indiscernible sequence. To verify that $T$ does not have the independence property, it suffices to show that if $\varphi(x, \bar{b})$ is any formula with parameters $\bar{b} \subseteq M$ then

$$
\left\{i \in \omega: M \models \varphi\left(a_{i}, \bar{b}\right)\right\}
$$

is either finite or cofinite (see [14]). By the fact that o-minimal theories do not have the independence property (see [15]) and quantifier elimination, we are reduced to considering formulas of the form $H(\lambda x+c)$ and $V(\lambda x+c)$. Here $c$ is an arbitrary element of $M$. We focus on the first of these; the second is similar. Suppose that for some $i<j \in \omega$ we have that

$$
M \models H\left(\lambda a_{i}+c\right) \wedge H\left(\lambda a_{j}+c\right) .
$$

Note that this is equivalent to saying that $a_{i}, a_{j}$ and $-\frac{c}{\lambda}$ are all in the same coset of the subgroup $H(M)$. Thus by indiscernibility we must have that for any $k \in \omega, a_{k}$ and $a_{i}$ are in the same coset of $U(M)$. Thus $a_{k}$ must be in the same coset as $-\frac{c}{\lambda}$ and hence

$$
M \models H\left(\lambda a_{k}+c\right) .
$$

Thus if the set

$$
\left\{i \in \omega: M \models H\left(\lambda a_{i}+c\right)\right\}
$$


has at least two elements, it is the entirety of $\omega$.

Finally we point out that the bounded PRC fields provide another class of examples of ordered structures with the exchange property (see [13]), although they do not appear to have the desired constructibility properties of the examples given here.

\section{References}

[1] Baisalov, Y., and B. Poizat, "Paires de structures o-minimales," The Journal of Symbolic Logic, vol. 63 (1998), pp. 570-78. Zbl 0910.03025. MR 1627306. 283, 285, 286

[2] Chatzidakis, Z., and A. Pillay, "Generic structures and simple theories," Annals of Pure and Applied Logic, vol. 95 (1998), pp. 71-92. Zbl 0929.03043. MR 1650667. 287, 288, 289

[3] Cherlin, G., and M. A. Dickmann, "Real closed rings. II. Model theory," Annals of Pure and Applied Logic, vol. 25 (1983), pp. 213-31. Zbl 0538.03028. MR 730855. 287

[4] Dolich, A., C. Miller, and C. Steinhorn, "O-minimal open cores," in preparation. 286

[5] Dougherty, R., and C. Miller, "Definable Boolean combinations of open sets are Boolean combinations of open definable sets," Illinois Journal of Mathematics, vol. 45 (2001), pp. 1347-50. Zbl 0991.54006. MR 1895461. 286

[6] van den Dries, L., "T-convexity and tame extensions. II," The Journal of Symbolic Logic, vol. 62 (1997), pp. 14-34. Zbl 0922.03055. MR 1450511. 287

[7] van den Dries, L., and A. H. Lewenberg, “T-convexity and tame extensions," The Journal of Symbolic Logic, vol. 60 (1995), pp. 74-102. Zbl 0856.03028. MR 1324502. 287, 288

[8] Macpherson, D., D. Marker, and C. Steinhorn, "Weakly o-minimal structures and real closed fields," Transactions of the American Mathematical Society, vol. 352 (2000), pp. 5435-83 (electronic). Zbl 0982.03021. MR 1781273. 281, 282, 283, 286

[9] Marker, D., “Omitting types in $\mathcal{O}$-minimal theories," The Journal of Symbolic Logic, vol. 51 (1986), pp. 63-74. Zbl 0596.03031. MR 830073. 285

[10] Mellor, T., "Imaginaries in real closed valued fields I," preprint available at http://www.uni-regensburg.de/Fakultaeten/nat_Fak_I/RAAG/preprints/0074.html. 287, 288

[11] Miller, C., "Expansions of dense linear orders with the intermediate value property," The Journal of Symbolic Logic, vol. 66 (2001), pp. 1783-90. Zbl 1001.03039. MR 1877021. 282,286

[12] Miller, C., and P. Speissegger, "Expansions of the real line by open sets: Ominimality and open cores," Fundamenta Mathematicae, vol. 162 (1999), pp. 193-208. Zbl 0946.03045. MR 1736360. 286

[13] Onshuus, A., "th-forking, algebraic independence and examples of rosy theories," preprint available at http://arxiv.org/abs/math.LO/0306003. 291

[14] Pillay, A., An Introduction to Stability Theory, vol. 8 of Oxford Logic Guides, The Clarendon Press, New York, 1983. Zbl 0526.03014. MR 719195. 290 
[15] Pillay, A., and C. Steinhorn, "Definable sets in ordered structures. I," Transactions of the American Mathematical Society, vol. 295 (1986), pp. 565-92. Zbl 0662.03023. MR 833697. 281, 282, 290

\section{Acknowledgments}

We thank the anonymous referee for many helpful suggestions.

Department of Mathematics, Statistics, and Computer Science

University of Illinois at Chicago

851 S Morgan St (M/C 249)

Chicago IL 60607

adolich@math.uic.edu 